\title{
Rhythmic Feedback Control of a Blind Planar Juggler
}

\author{
Renaud Ronsse, Student Member, IEEE, Philippe Lefèvre, Member, IEEE, and Rodolphe Sepulchre, Member, IEEE
}

\begin{abstract}
The paper considers the feedback stabilization of periodic orbits in a planar juggler. The juggler is "blind," i.e, he has no other sensing capabilities than the detection of impact times. The robustness analysis of the proposed control suggests that the arms acceleration at impact is a crucial design parameter even though it plays no role in the stability analysis. Analytical results and convergence proofs are provided for a simplified model of the juggler. The control law is then adapted to a more accurate model and validated in an experimental setup.
\end{abstract}

Index Terms-Bouncing ball, impact dynamics, intermittent control, juggling.

\section{INTRODUCTION}

B REATHING, chewing, juggling, walking, hopping, and running are rhythmic tasks that are routinely accomplished by humans and animals. Many recent studies in neuroscience have focused on the distinct features involved in the motor control of rhythmic tasks, either at low-level circuitry (central patten generators; e.g., [1], [2]) or high-level brain recruitment (e.g., [3]). As emphasized in a recent paper by Schaal and Schweighofer [4], questions in motor control often parallel questions in robotics, and a fruitful interaction has emerged between neuroscience and engineering. This is particularly true for the control of rhythmic tasks that involve an intermittent contact between the controlled body and its environment: in a juggling task, the continuous-time motion of the actuator is used to control the continuous-time motion of an object but the contact between the actuator and the juggled object is only intermittent, and, in fact, purely impulsive in the limit of impact juggling. Simple juggling models have served as benchmark models in many studies in engineering (modeling, dynamics, and con-

Manuscript received July 10, 2006; revised December 12, 2006. This paper was recommended for publication by Associate Editor Hirai and Editor K. Lynch upon evaluation of the reviewers' comments. This paper presents the research results of the Belgian Network Dynamical Systems, Control, and Optimization funded by the Interuniversity Attraction Poles Programme, initiated by the Belgian State, Science Policy Office. The scientific responsibility rests with its author(s). This work was supported by Grants "Fonds National de la Recherche Scientifique," the "Fondation pour la Recherche Scientifique Médicale," and the "Fonds Spéciaux de Recherche" by the Université Catholique de Louvain. This paper was presented in part at the 44th IEEE Conference on Decision and Control and European Control Conference, Seville, Spain, and in part at the 45th IEEE Conference on Decision and Control, San Diego, CA, 2006.

R. Ronsse and R. Sepulchre are with the Department of Electrical Engineering and Computer Science, Université de Liège, Liège, Belgium (e-mail: R. Ronsse@ulg.ac.be; R. Sepulchre@ulg.ac.be).

P. Lefèvre is with the Center for Systems Engineering and Applied Mechanics (CESAME), Université Catholique de Louvain, Belgium (e-mail: lefevre@csam.ucl.ac.be).

This paper has supplementary downloadable material available at http:// ieexplore.ieee.org, provided by the authors. This movie is a wmv file illustrating the stabilization of impact juggling orbits with the "Wiper" robot. It has been recorded at the Université catholique de Louvain, Belgium, and analyzed at the Université de Liège, Belgium. The metronome beeps are triggered by the accelerometers when they detect an impact. This illustrates proper impact detection. This material is $15.9 \mathrm{MB}$ in size.

Digital Object Identifier 10.1109/TRO.2007.900602 trol [5]-[15]) as well as in experimental studies in neuroscience [16]-[18]. Juggling is indeed a rhythmic yet confined task that involves inter-limb coordination, eye-hand coordination (sensorimotor mapping), and phase-locking relationships between these effectors and the juggled objects [19]. Juggling models have often provided a theoretical basis for the design of more complex rhythmic tasks such as the locomotion control of multipod robots (see, e.g., [20] or [21] for a recent review) or biped walking (see, e.g., the RABBIT project, as described in [22]).

The paper presents an experimental validation of rhythmic pattern control on a planar juggler, schematized in Fig. 1(a): a ball moves in a 2-D plane under the gravitational field, and is impacted by two independent arms rotating around their fixed intersection. The continuous-time actuators periodically impact the ball whose dynamics are only governed by gravity, between two impacts. This planar juggler possesses several periodic orbits, the simplest one being the period-one and the period-two, that mimic the popular shower juggling pattern (Fig. 1). Stabilization of these periodic orbits requires frequency-locked actuation of the edges, while their phase relationship determines the shape of the orbit, which is stabilized. Such phase relationships between the effectors in coordinated movements are of prime interest to study both the shape and the stability of the patterns. For example, in locomotion, gait shifts are induced by phase transitions in the effectors movements. Moreover, our juggling paradigm led to handy mathematical modeling since the impacts are instantaneous.

In human juggling experiments, it has long been recognized that the control of timing is ubiquitous, either for throwing and catching time in the three-balls cascade [23] or for 1-D ball bouncing [17]. This has been further emphasized recently in a robotic experiment by Hirai and Miyazaki [15]. They studied a juggling-like ball-passing task in the horizontal plane (i.e., no effect of gravity) whose stabilization is based only on feedback measurement of the impact times between the balls and the robot. The objective of the present paper is to emphasize the role of impact times as a central information for the model-based feedback control of rhythmic tasks and to demonstrate the existence of robust control schemes based on this sole discrete information. We succeeded in robustly stabilizing a period-two orbit [Fig. 1(c)], with a control law that is based on a state observer whose sole input is the impact times detection. The measurement of the occurrences of timed events, like the impacts in our task, could obviously be generalized as an exclusive source of feedback for most rhythmic systems. For example, in locomotion, the initiation and/or termination of the stance phase are such marked periodic events in the cycle that could be useful for feedback.

In [24], we provided experimental validation of a sensorless control law (a purely sinusoidal actuation of the juggler arms) that robustly stabilizes the period-one orbit [Fig. 1(b)], but failed to robustly stabilize the period-two orbit. The control 


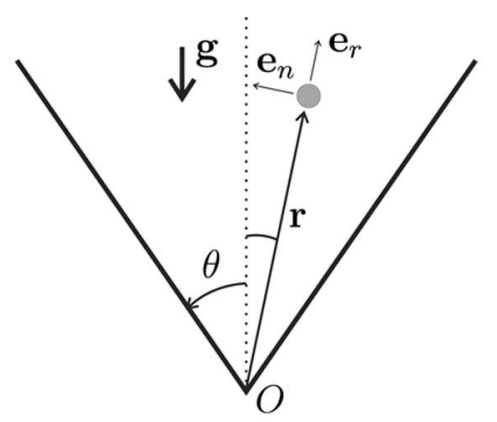

(a) (b)

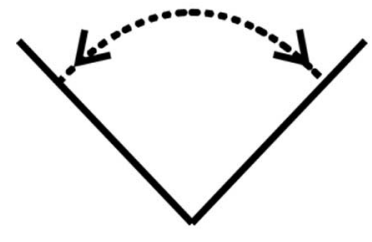

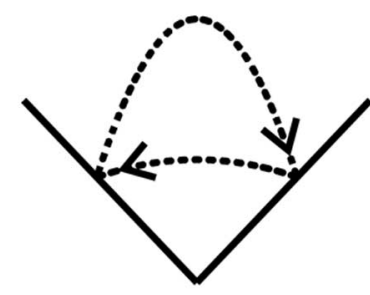

(c)
Fig. 1. Wedge billiard and two of its periodic orbits. (a) Wedge billiard. (b) Period-One orbit. (c) Period-Two orbit.

law proposed in the present paper can be interpreted as a phase and amplitude modulation of the sinusoidal control law, based on the feedback information provided by the impact times.

The experimental results of the paper are supported by a theoretical analysis, which investigates both the stability and the robustness of the control design. Exploiting a special configuration of the juggler in which the dynamical model is considerably simplified, the theoretical analysis is developed on the celebrated dynamics of a ball vertically bouncing on a vibrating table. This simplified model allows for analytical developments and facilitates the comparison of our approach with abundant literature [8]-[10], [25]-[29]. We use the continuous-time actuation of the table to stabilize a reference trajectory for the bouncing ball. The reference trajectory is discrete, i.e., it assigns the state of the bouncing ball at successive impacts. We first derive a controller that achieves deadbeat tracking of an arbitrary reference trajectory. This controller is a state feedback controller, which is then turned into an output feedback controller: the state of the ball is reconstructed from the sole impact times by means of a deadbeat observer.

The output feedback controller assigns the impact position and the impact velocity of the actuator, but the stability of the closed-loop system puts no constraints on the impact acceleration. In contrast, this parameter turns to be a crucial design parameter in the robustness analysis of the closed-loop system. We focus the robustness analysis on the model uncertainty arising from the impact model. The design is based on the simple Newton's impact model, and we model the uncertainty by treating the variations of the coefficient of restitution as an external disturbance. Analyzing the transfer function from this disturbance to the impact velocity, we show that the dynamics can be exactly canceled by a proper choice of the impact acceleration. This particular tuning requires negative impact acceleration, and turns out to be a key of success in the experimental validation
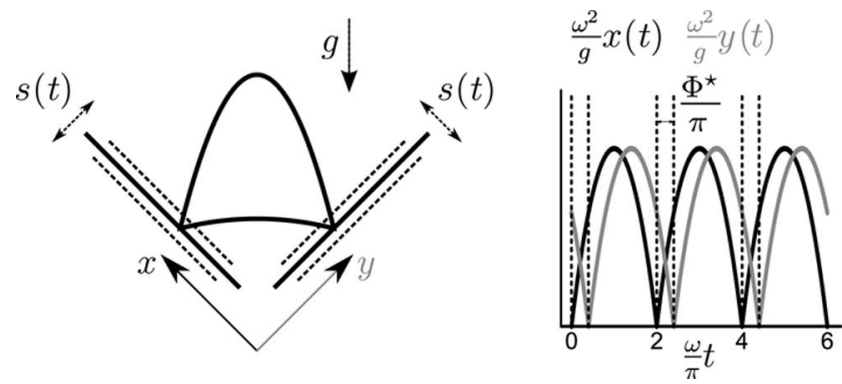

Fig. 2. Special configuration of a planar juggler that decouples the 2-D ball motion (left) into two independent 1-D bouncing ball motions (right). The right frame represents the nondimensional ball dynamics along the $x$ and $y$ axes, over nondimensional time.

of our control law. It must be emphasized that our experimental validation suffers from large discrepancies between the model and the real setup dynamics. For example, the Newton impact model does not capture the spin effect of the puck, which is clearly visible in the period-two pattern. However, the focus of our approach is precisely to validate the robustness of a model-based control scheme in a real setup in spite of the many discrepancies between the model and the experimental setup.

The negative acceleration is in agreement with the sensorless sinusoidal actuation-that stabilizes the periodic pattern with negative acceleration at impact. It is in contrast with the mirror law [25]-[27] that generates positive acceleration at impact. The role of a negative impact acceleration was also emphasized in previous results by Sternad and colleagues [16]-[18] who studied humans juggling a 1-D bouncing ball. In steady state, experienced human jugglers also impact the ball with a decelerating racket. This suggests that humans do recruit robust control schemes in order to rest on minimum feedback demand. In more complicated juggling tasks, e.g., the three balls cascade, it is also demonstrated that the visual attention is kept focused to a limited zone of the cycle, i.e., the apex, in order to extract as much feedback information as possible with limited demand [30].

The rest of this paper is organized as follows. Section II introduces the mathematical problem statement. Section III derives the basic output feedback control law for the bouncing ball dynamics. In particular, Section III-E describes an observer that reconstructs the state only from impact times measurement. Section IV discusses the robustness of this control law. Section $\mathrm{V}$ presents an experimental validation of this control law in a real experimental setup: the Wiper robot. Some technical developments are detailed in the Appendixes.

\section{Problem Statement}

For the mathematical developments of the paper, we consider a special configuration of the planar juggler, illustrated in Fig. 2: the two edges are assumed to remain aligned with the two orthogonal axes of a fixed reference frame (this requires parallel actuation of the edges, in contrast with the rotational actuation of the Wiper robot studied in Section V).

The resulting dynamics nicely decouple with this special configuration: the 2-D motion of the juggled ball projects on each 


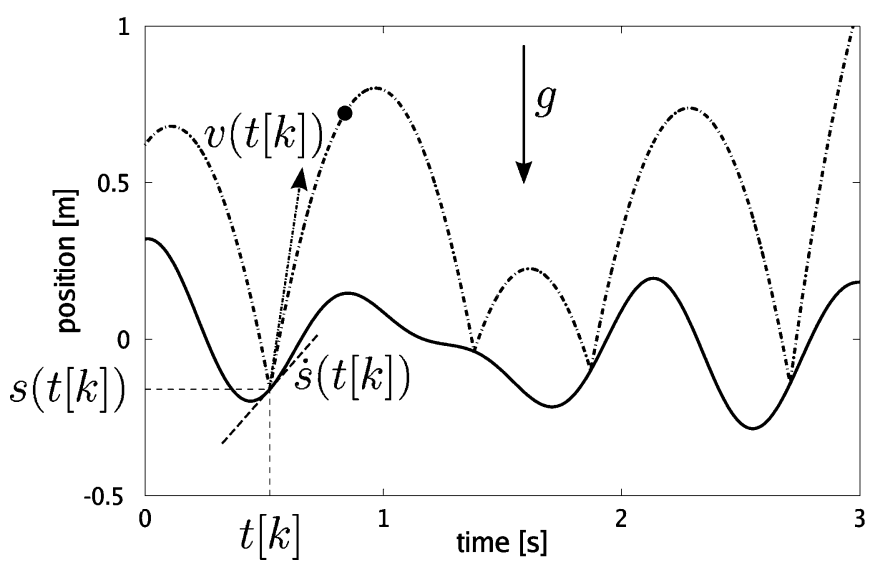

Fig. 3. 1-D bouncing ball. The actuator (respectively the ball) trajectory is depicted with solid (respectively dash-dotted) lines over time. At time $t[k](k \mathrm{th}$ impact), the actuator (and ball) position is $s(t[k])$, the actuator velocity is $\dot{s}(t[k])$ and the ball postimpact velocity is $v(t[k])$.

axis to a 1-D motion that is unaffected by the bounces on the other axis [11]. The dynamics along each axis are the dynamics of a 1-D bouncing ball.

A period-two orbit of the planar juggler projects onto a periodone orbit along each axis. The phase shift $\Phi^{*}$ between the $x$ orbit and the $y$-orbit controls the shape (i.e., the apex difference between the low toss and the high toss) of the period-two planar orbit, since the impact times obey

$$
\begin{aligned}
t\left[k_{x}\right] & =\frac{2 \pi k_{x}}{\omega} \\
t\left[k_{y}\right] & =\frac{2 \pi k_{y}+\Phi^{*}}{\omega}
\end{aligned}
$$

where $k_{x}$ and $k_{y}$ denote the $k$ th impact along the $x$ and $y$ axes, respectively, and $\omega$ denotes the cycle frequency.

Based upon this analogy, the stabilization of the period-two planar orbit of the juggler reduces to the stabilization of a periodone orbit in two independent 1-D bouncing balls. As a consequence, the design of a feedback control is first studied on the simple 1-D bouncing ball model. The extension of the design to a more general planar juggler model is postponed to Section $\mathrm{V}$.

In the 1-D bouncing ball dynamics [5], [6], the actuation is the continuous-time motion $s(t)$. The ball dynamics are governed by the gravitational field $g$ and the impacts with the actuator.

Between the $(k)$ th and the $(k+1)$ th impacts, occurring at time $t[k]$ and $t[k+1]$, the ball follows a parabolic trajectory (see Fig. 3). The position of impact, therefore, obeys the following discrete-time flight map, derived from the ball motion:

$$
\begin{aligned}
s(t[k+1])=s(t[k])+v(t[k])(t[k+1]) & -t[k]) \\
& \left.-\frac{g}{2}(t[k+1])-t[k]\right)^{2}
\end{aligned}
$$

where $v(t[k])$ is the postimpact velocity and $g$ the gravitational field. Similarly, the preimpact velocity, $v^{-}(t[k+1])$ is equal to

$$
v^{-}(t[k+1])=v(t[k])-g(t[k+1]-t[k]) .
$$

Based on Newton's law, the relative velocity of the ball with respect to the actuator is reversed at impact and multiplied by the coefficient of restitution $0 \leq e \leq 1$ that models the energy dissipation

$$
v(t[k+1])-\dot{s}(t[k+1])=-e\left(v^{-}(t[k+1])-\dot{s}(t[k+1])\right) .
$$

Equation (3) assumes that the actuator motion is unaffected by the impacts. This assumption is valid if the actuator is largely heavier than the ball.

The complete bouncing ball dynamics are, therefore, described by the discrete Poincaré map, whose state is the impact position $s[k]=s(t[k])$ and postvelocity $v[k]=v(t[k])$

$$
\begin{aligned}
s[k+1]= & s[k]+v[k](t[k+1])-t[k]) \\
& \left.-\frac{g}{2}(t[k+1])-t[k]\right)^{2} \\
v[k+1]= & -\operatorname{ev}[k]+\operatorname{eg}(t[k+1]-t[k]) \\
& +(1+e) \dot{s}[k+1]
\end{aligned}
$$

where $\dot{s}[k]=\dot{s}(t[k])$. Equation (4) is the flight map and (5) is the impact rule, derived from (2) and (3).

The flight time is deduced from (4) as

$t[k+1]-t[k]=\frac{v[k]+\sqrt{v[k]^{2}-2 g(s[k+1]-s[k])}}{g}$.

Given a reference trajectory $\left(s_{\rho}[\bullet], v_{\rho}[\bullet]\right)$ for the system (4) and (5), we study the design of a continuous-time motion $s(t)$ that achieves asymptotic tracking of this discrete reference.

\section{FeEdBACK Control of the Bouncing BALL}

\section{A. Two Popular Control Laws Revisited}

In this section, we revisit two basic algorithms that have been reported in the literature to stabilize the period-one pattern of the bouncing ball.

This particular reference trajectory is the fixed point of (4) and (5)

$$
\begin{aligned}
v^{*} & =\frac{g}{2} \Delta t^{*} \\
\dot{s}^{*} & =\frac{1-e}{1+e} \frac{g}{2} \Delta t^{*}
\end{aligned}
$$

where $\Delta t^{*}$ is the steady-state flight time. The steady-state impact position $s^{*}$ is not assigned by the steady-state equations, as long as $s(t)$ satisfies (8).

The first proposed "control" law is the sinusoid $s(t)=$ $A \sin (\omega t)$ [5], [6]. This control does not require any sensing, and forces the steady-state flight time to

$$
\Delta t^{*}=n \frac{2 \pi}{\omega}
$$

where $n=1$ when there is one actuator period between two successive impacts.

The amplitude $A$ and frequency $\omega$ can be tuned to match an arbitrary steady state $\left(s^{*}, v^{*}\right)$. The period-one motion stability is studied via the linearization of (4) and (5) (see Appendix I and [31]). It is easily shown that stability of the orbit implies that the actuator acceleration at impact is negative, since the stability 

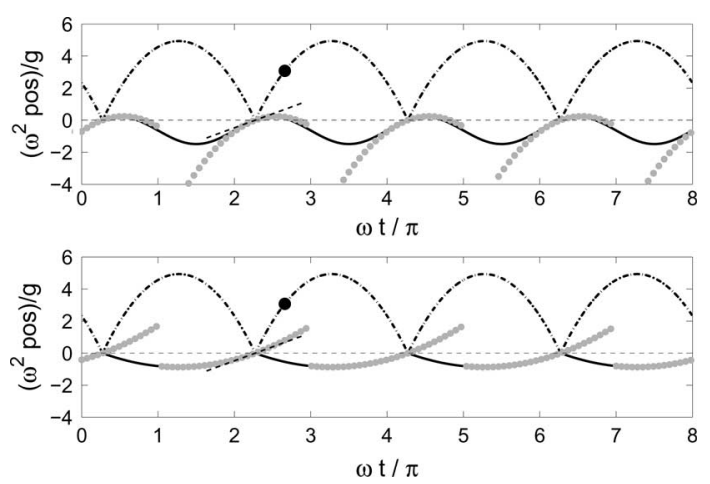

Fig. 4. Comparison of the sinusoidal trajectory (top) and the mirror law (bottom) to stabilize the period-one pattern. The actuator (resp. the ball) nondimensional position is depicted with solid (resp. dash-dotted) lines over nondimensional time. The gray dots denote the piecewise quadratic trajectory that matches the actuator position, velocity, and acceleration at impact; see Section III-C.

condition (54) defines the steady-state acceleration range as

$$
\frac{-2\left(1+e^{2}\right)}{(1+e)^{2}} g<\ddot{s}^{*}<0 .
$$

This is illustrated in Fig. 4 (top).

In contrast with the sensorless sinusoidal law, the mirror law [25]-[27] is a feedback strategy based upon permanent tracking of the ball. For simplicity, we focus on the 1-D version of this control law, but it has been successfully implemented in 2-D and 3-D juggling robots

$$
s(t)=\frac{-(1-e)}{1+e} \beta(t)-\kappa_{1}\left(E_{\rho}^{*}-E(t)\right) \beta(t) .
$$

In (11), $\beta(t)$ and $E(t)$ denote the ball position and energy at time $t$. The first term of (11) is mirroring the ball trajectory; the second one is a proportional feedback that is used to isolate a particular period-one pattern, characterized by its energy level: $E_{\rho}^{*}=$ $g s_{\rho}^{*}+0.5\left(v_{\rho}^{*}\right)^{2}$. The gain $\kappa_{1}$ will determine the pole placement of the closed-loop system. In steady state, that is when $E(t)=$ $E_{\rho}^{*}$, the mirror law behavior is depicted in Fig. 4 (bottom).

Comparing the sinusoidal law and the mirror law in Fig. 4, we see that both of them stabilize the bouncing ball period-one at the same steady state $\left(s^{*}, v^{*}\right)$, but with significant differences in the underlying continuous-time control law. We observe that the sinusoidal trajectory is decelerating at impact while the mirror law is accelerating: the acceleration of the steady-state mirror law is $(1-e) /(1+e) g \geq 0$.

The two control strategies that stabilize the same pattern are, thus, clearly distinct. The first one is sensorless while the second requires a permanent tracking of the ball, which suggests that the acceleration at impact possibly influences the feedback requirements of the control law.

\section{B. Stabilization With Sensorless Sinusoidal Actuation}

Even if there is no control loop with the sensorless sinusoidal actuation $s(t)=A \sin (\omega t)$, it is of interest to interpret its stabilizing feedback mechanism in the system dynamics.

The linearized dynamics [see (48) in Appendix I] admit the state-space representation

$$
\begin{aligned}
\mathbf{x}[k+1] & =\mathbf{A} \mathbf{x}[k]+\mathbf{B}(A, \omega) u[k] \\
y[k] & =t[k+1]=\mathbf{C x}[k]
\end{aligned}
$$

where the "controller input" equals the "output": $u[k]=y[k]$, i.e., the impact time $t[k+1]$. This state-space representation is both controllable and observable.

In this representation, the sinusoidal actuation is interpreted as a proportional feedback of the output $t[k+1]$, emphasizing the importance of estimating the next impact time for stabilization. The first element of the matrix $\mathbf{B}$ is a feedback gain equal to the actuator acceleration at impact. Negative feedback, thus, requires a negative acceleration. A similar observation has been proposed for the 2-D wedge billiard [32].

\section{State Feedback Control}

This section describes a more general class of controllers aiming at tracking a time-varying referenced trajectory, impact after impact. Given the flight time equation (6), exact matching between the real and the referenced next impact position

$$
s[k+1]=s_{\rho}[k+1]
$$

is provided, if the next impact occurs at time

$$
t_{u}[k+1]=t[k]+\frac{v[k]+\sqrt{v[k]^{2}-2 g\left(s_{\rho}[k+1]-s[k]\right)}}{g} .
$$

Given (5) and (15), the impactor velocity at impact must be equal to

$$
\dot{s}_{u}[k+1]=\frac{v_{\rho}[k+1]-e \sqrt{v[k]^{2}-2 g\left(s_{\rho}[k+1]-s[k]\right)}}{1+e}
$$

in order to provide $v[k+1]=v_{\rho}[k+1]$.

As illustrated in Fig. 4, the two control laws described in Section III-A achieve the same stabilization objective, but differ in the impact acceleration. In order to illustrate how the actuator acceleration at impact influences the robustness of the feedback system, we consider a more general family of control laws in the form of a piecewise quadratic function of time, reinitialized after each impact

$$
\begin{aligned}
s(t)=s_{u}[k+1]+\dot{s}_{u}[k+1]\left(t-t_{u}\right. & {[k+1]) } \\
& +\frac{\gamma}{2}\left(t-t_{u}[k+1]\right)^{2}
\end{aligned}
$$

$$
\begin{gathered}
s[k+1]=s_{\rho}[k+1]+\frac{v_{\rho}[k+1]-e \sqrt{v[k]^{2}-2 g\left(s_{\rho}[k+1]-s[k]\right)}}{1+e}\left(t[k+1]-t[k]-\frac{v[k]+\sqrt{v[k]^{2}-2 g\left(s_{\rho}[k+1]-s[k]\right)}}{g}\right) \\
+\frac{\gamma}{2}\left(t[k+1]-t[k]-\frac{v[k]+\sqrt{v[k]^{2}-2 g\left(s_{\rho}[k+1]-s[k]\right)}}{g}\right)^{2}
\end{gathered}
$$




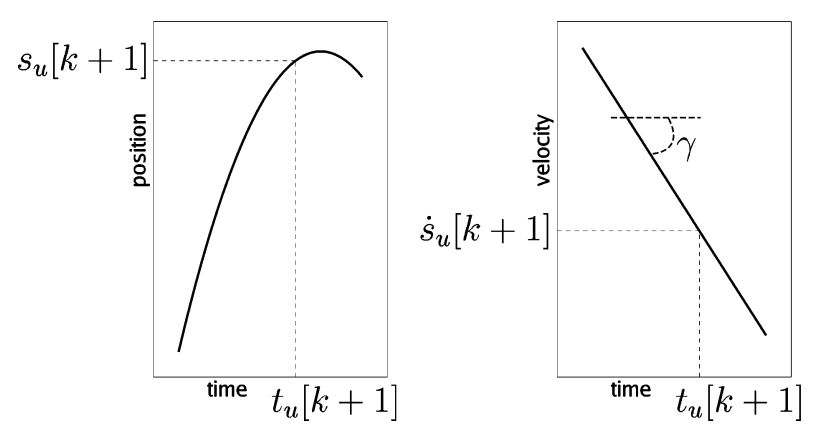

Fig. 5. Local position (left) and velocity (right) profiles of the actuator trajectory. The position profile is given by (17) while the velocity is its first derivative; $\gamma$ is the acceleration.

for $t[k]<t \leq t[k+1], \gamma$ denoting the actuator acceleration. At time $t=t_{u}[k+1]$, the actuator position (velocity, resp.) is equal to $s_{u}[k+1]\left(\dot{s}_{u}[k+1]\right.$, resp.); see Fig. 5. The gray dots on Fig. 4 illustrate the steady-state behavior of this control law when the parameters are tuned to match the ball position, velocity, and acceleration at impact with the two control laws presented in Section III-A.

The quadratic parameterization of the control (17) is convenient to obtain an explicit expression of the impact times: $t[k+1]$ is indeed the solution of (4) and (17) at time $t=$ $t[k+1]$, which defines a second-order polynomial in $t$. The controller inputs are $t_{u}[k+1](15), s_{u}[k+1]=s_{\rho}[k+1]$, and $\dot{s}_{u}[k+1](16)$ and are functions of the state $(s[k], v[k])$ and impact time $t[k]$. Deadbeat convergence of this tracking control law, i.e., convergence of the solution to the reference trajectory after a finite number of time steps, is established in Section III-D.

\section{Deadbeat Convergence}

Substituting (14), (15), and (16) into (17) at time $t=t[k+$ 1 ], we obtain (18), given at the bottom of the previous page. Substituting (6) into (18), we obtain a second-order polynomial in $t[k+1]$, whose positive root is

$$
t[k+1]=t[k]+\frac{v[k]+\sqrt{v[k]^{2}-2 g\left(s_{\rho}[k+1]-s[k]\right)}}{g} .
$$

Comparing this with (6) yields

$$
s[k+1]=s_{\rho}[k+1]
$$

and therefore

$$
\begin{aligned}
v[k+1] & =v_{\rho}[k+1] \\
t[k+1] & =t_{u}[k+1]
\end{aligned}
$$

reflecting that the positive solution of (4), (5), and (17) is the exact matching between the reference and the real impact state.

To summarize, the continuous-time control law determined by the quadratic expression (17) and the discrete control (14)(16) ensures deadbeat convergence of the impact state after one time step. So far, the acceleration $\gamma$ in (17) is a free parameter and does not influence the convergence. The control law is a tracking controller, i.e., the reference trajectory $\left(s_{\rho}[\bullet], v_{\rho}[\bullet]\right)$ is arbitrary, as long as it corresponds to a solution of the dynamical system (4) and (5).

\section{E. Output Feedback Deadbeat Control}

The piecewise quadratic control proposed in Section III-C uses the full state $(s[k], v[k])$ of the system (4) and (5). To reduce the sensing requirements of the controller, we now assume that only the continuous-time actuator motion $s(t)$ is measured, together with the impact times $t[k]$ : they provide the impact position $s[k]$ and velocity $\dot{s}[k]$. In this section, we derive a deadbeat observer that reconstructs the postimpact ball velocity $v[k]$ from the measured impact times $t[k]$ and actuator motion $s(t)$.

Postimpact velocity is estimated by an observer that is a copy of the velocity dynamics (5)

$$
\hat{v}[k]=-e v[k-1]+e g(t[k]-t[k-1])+(1+e) \dot{s}[k]
$$

while $v[k-1]$ is obtained from (4)

$$
\begin{aligned}
\hat{v}[k]= & -e\left(\frac{s[k]-s[k-1]}{t[k]-t[k-1]}+\frac{g}{2}(t[k]-t[k-1])\right) \\
& +e g(t[k]-t[k-1])+(1+e) \dot{s}[k] \\
= & e \frac{g}{2}(t[k]-t[k-1])-e \frac{s[k]-s[k-1]}{t[k]-t[k-1]}+(1+e) \dot{s}[k] .
\end{aligned}
$$

Equation (24) defines a deadbeat velocity observer using the impact times as sole input in addition to the actuator motion. Deadbeat convergence is ensured in one time step since $\hat{v}[k]=$ $v[k], \forall k>1$.

The output feedback controller, whose only measured signals are the impact times, is then obtained by replacing the actual state variable $v[k]$ by the estimated variable $\hat{v}[k]$ in (14)-(16). Its deadbeat convergence is established in the following proposition:

Proposition 1: (Deadbeat convergence of the piecewise quadratic output feedback controller) Consider the bouncing ball dynamics (4) and (5) and a reference trajectory $\left(s_{\rho}[k], v_{\rho}[k]\right), k \geq 0$. The output feedback control

$$
\begin{aligned}
& s(t)=s_{\rho}[k+1]+\dot{s}_{u}[k+1]\left(t-t_{u}[k+1]\right) \\
& +\frac{\gamma}{2}\left(t-t_{u}[k+1]\right)^{2}, t[k]<t \leq t[k+1] \\
& t_{u}[k+1]=t[k]+\frac{\hat{v}[k]+\sqrt{\hat{v}[k]^{2}-2 g\left(s_{\rho}[k+1]-s[k]\right)}}{g} \\
& \dot{s}_{u}[k+1]=\frac{v_{\rho}[k+1]-e \sqrt{\hat{v}[k]^{2}-2 g\left(s_{\rho}[k+1]-s[k]\right)}}{1+e} \\
& \hat{v}[k]=e \frac{g}{2}(t[k]-t[k-1])-e \frac{s[k]-s[k-1]}{t[k]-t[k-1]} \\
& +(1+e) \dot{s}[k]
\end{aligned}
$$

ensures deadbeat convergence of the 1-D bouncing ball state toward the reference $\left(s_{\rho}[k], v_{\rho}[k]\right)$ after two impacts (i.e., $k>2$ ).

Proof: The first impact is required to ensure convergence of the observer, since $\hat{v}[k]=v[k], \forall k>1$. As soon as $\hat{v}[k]=v[k]$, a second impact is required to achieve the deadbeat convergence of the controller, as shown in Section III-D. 


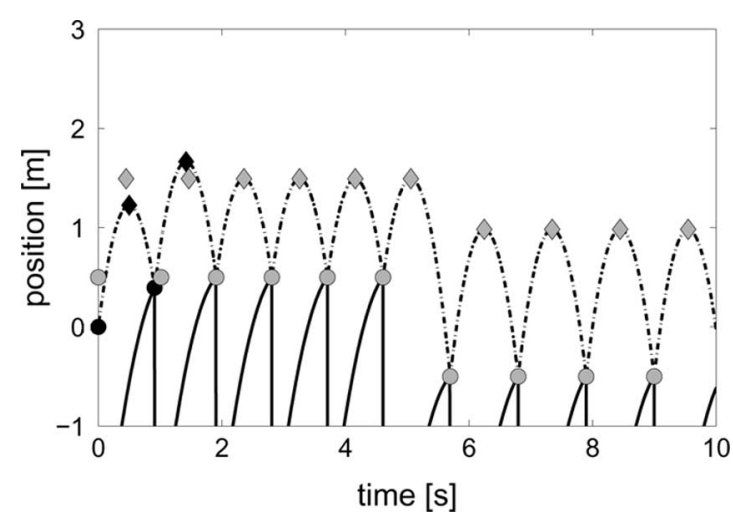

Fig. 6. Output feedback control of the bouncing ball with the control law (25). The actuator (resp. the ball) position is depicted with solid (resp. dash-dotted) lines over time. Actual impacts position (resp. apex position) are represented with black circles (resp. black diamonds). Reference positions are accordingly represented with gray markers. $e=0.7, \gamma<0$.

The deadbeat convergence is illustrated in Fig. 6. At the third impact, both the reference impact position and the reference apex (which is an image of the reference postimpact velocity) are reached. Fig. 6 illustrates the proper tracking of time-varying references since both the position and velocity references change at the seventh impact.

\section{F. Blind Mirror Law Based on Output Feedback}

Assuming a ballistic flight between two impacts, the mirror law (11) can also be adapted to an output feedback control, with the impact times as measured output

$$
s(t)=\left(\frac{-(1-e)}{1+e}-\kappa_{1}\left(E_{\rho}^{*}-\hat{E}(t)\right)\right) \hat{\beta}(t)
$$

since both the ball position and energy can be estimated from impact state

$$
\begin{aligned}
& \hat{\beta}(t)=s[k]+\hat{v}[k](t-t[k])-\frac{g}{2}(t-t[k])^{2} \\
& \hat{E}(t)=\hat{E}[k]=g s[k]+\frac{1}{2} \hat{v}[k]^{2}
\end{aligned}
$$

for $t[k] \leq t<t[k+1]$. The tracking mirror law (11) requires a permanent tracking of the ball as sensory input. In contrast, the blind mirror law (26) based on output feedback only uses impact times and reconstructs the postimpact velocity $v[k]$ via the observer described in Section III-E.

Since both the piecewise quadratic law (25) and the blind mirror law (26) require the same sensing capabilities, their robustness will be compared in Section IV.

\section{RoBUSTness to MODEL UnCERTAINTIES}

The acceleration parameter $\gamma$ appearing in (25) played no role in the stability and convergence analysis. This section stresses the importance of this parameter for robustness purposes. We show that particular negative accelerations efficiently optimize either static or dynamical perturbations induced by a poor estimate of the coefficient of restitution $e$.

\section{A. Uncertainty of the Impact Model}

Among the several sources of uncertainty of the model (4) and (5), the impact model (3) is probably central. While the Newton impact law models $e$ as a constant, this parameter is varying in experimental conditions. Furthermore, the linear relationship between the pre- and postimpact velocities (3) is certainly not respected outside a narrow range of impact velocities.

We model the uncertainty on the coefficient of restitution $e$ by considering the perturbed impact rule

$$
\begin{aligned}
v[k+1]-\dot{s}[k+1]=-(e+\Delta e[k+1])\left(v^{-}\right. & {[k+1] } \\
& -\dot{s}[k+1])
\end{aligned}
$$

where $\Delta e[k+1]$ models the variation of the coefficient of restitution at time $t[k+1]$, w.r.t. the estimated value $e$. For the sake of simplicity, we study the robustness of the linearized feedback system, as derived in Appendix II.

From the linear state-space representation (50), we find the following closed-loop input-to-state transfer functions:

$$
\begin{aligned}
S(z) & =S_{\rho}(z)+\frac{2(1-e)}{1+e} \frac{1}{z} E(z) \\
V(z) & =V_{\rho}(z)+\frac{2}{1+e} \frac{z+\left(e^{2}+\frac{\gamma}{g}(1+e)^{2}\right)}{z} E(z)
\end{aligned}
$$

where $S(z), V(z), S_{\rho}(z), V_{\rho}(z)$, and $E(z)$ refer to the $z$-transforms of $g \delta s[k] /\left(v_{\rho}^{*}\right)^{2}, \delta v[k] / v_{\rho}^{*}, g \delta s_{\rho}[k] /\left(v_{\rho}^{*}\right)^{2}, \delta v_{\rho}[k] /$ $v_{\rho}^{*}$, and $\Delta e[k]$, respectively. In (28) and (29), the absence of dynamics in the transfer from references to states is due to the deadbeat convergence established in Section III-E. Section IV-B details the role of acceleration to reject the perturbations due to $\Delta e[k]$.

\section{B. Robustness Requires Negative Acceleration}

From (29), we see that the acceleration $\gamma$ can be designed to place the zero of the transfer function from $E(z)$ to $V(z)$. That design parameter will be discussed to optimize either the static or the dynamic performance.

1) Static Error: To let the postimpact velocity converge toward the reference $v_{\rho}[k]$, assuming a constant perturbation $\Delta e[k]=\Delta e$, one has to cancel the static gain of the transfer function from $E(z)$ to $V(z)$. This amounts to place the zero of (29) at $z=-1$, which requires the acceleration

$$
\gamma_{\text {stat }}=-\frac{1+e^{2}}{(1+e)^{2}} g .
$$

Interestingly, this optimal acceleration depends only on $e$, i.e., the estimated coefficient of restitution. Fig. 7 illustrates the behavior of the feedback system when the coefficient of restitution is estimated at $e=0.7$ while the real one is only $e+\Delta e=0.5$. The desired postimpact velocity is reached because the difference between impact and apex positions is the same for the reference as for the actual trajectory. It should be noticed, however, that a static error persists on the reference position: the static gain from $E(z)$ to $S(z)$ is indeed independent of $\gamma(28)$. This 


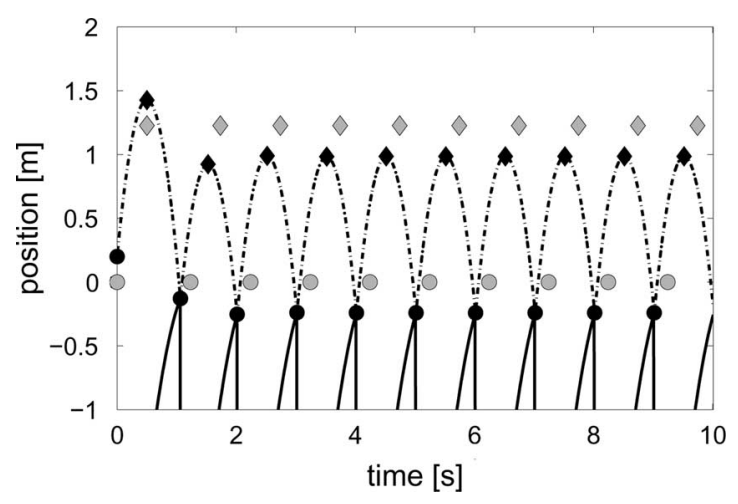

Fig. 7. Output feedback control of the bouncing ball with piecewise quadratic trajectory. The actuator (resp. ball) position is depicted with solid (resp. dashdotted) lines over time. Actual impacts position (resp. apex position) are denoted with black circles (resp. black diamonds). Reference positions are accordingly denoted by gray markers. $e+\Delta e=0.5, e=0.7, \gamma$ is given by (30).

static error does not appear to be detrimental to the robustness of the feedback system.

The optimal acceleration for static performance, as identified in (30), has also been derived from the original nonlinear equations [33].

It is of interest to relate this particular acceleration to the sinusoidal control discussed in Section III-A: the optimal acceleration (30) is exactly the middle point of the acceleration range where the period-one motion is stable (10).

2) Dynamic Performance: Robustness to a static error on $e$ is not the primary issue in real experiments because the average value of $e$ is easy to determine. In contrast, robustness is required against the sustained variability of $e$. By placing the zero of (29) at $z=0$, the dynamics from $E(z)$ to $V(z)$ are exactly cancelled:

$$
\gamma_{\mathrm{dyn}}=-\frac{e^{2}}{(1+e)^{2}} g .
$$

Interestingly, this optimal value closely matches the value that minimizes the numerically computed variability of the sinusoidally actuated bouncing ball in [17] and [18].

We summarize the robustness analysis of the piecewise quadratic law (25) with the following proposition.

Proposition 2: Consider the bouncing-ball dynamics (4) and (5) with a time-varying coefficient of restitution $e+\Delta e[k+1]$. Using the output feedback control (25), the transfer function from $\Delta e[k]$ to $\delta v[k] / v_{\rho}^{*}$ is given by

$$
\frac{V(z)}{E(z)}=\frac{2}{1+e} \frac{z+\left(e^{2}+\frac{\gamma}{g}(1+e)^{2}\right)}{z} .
$$

The choice $\gamma_{\text {stat }}=-\left(1+e^{2}\right) /(1+e)^{2} g$ (see (30)) ensures zero steady-state error while the choice $\gamma_{\mathrm{dyn}}=-e^{2} /(1+e)^{2} g$ (see (31)) cancels the transfer-function dynamics. Both choices result in negative acceleration at impact, with

$$
\gamma_{\text {stat }}<\gamma_{\text {dyn }} \leq 0
$$

3) Simulation Results: To illustrate the role of the impact acceleration for robustness in the nonlinear dynamics, we now compare the output piecewise quadratic controller (with the optimal negative accelerations previously identified) with the

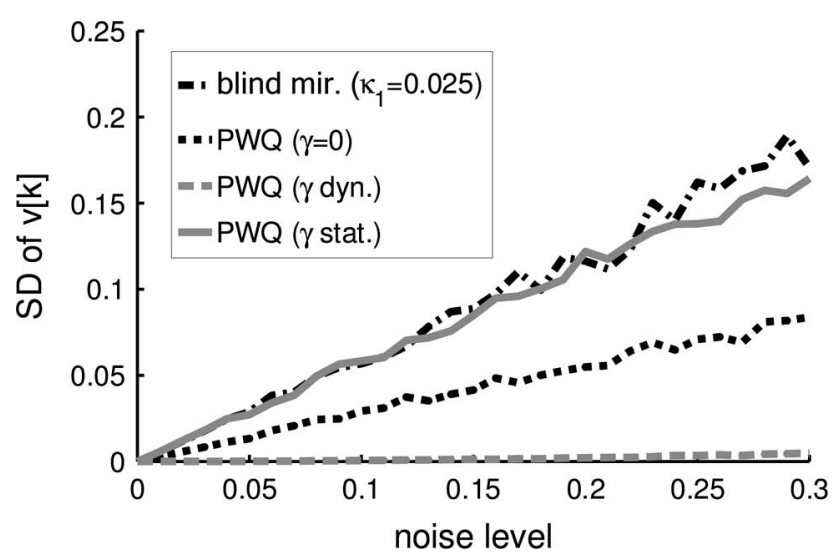

Fig. 8. Simulations of the nonlinear noisy bouncing ball dynamics with different control laws: the blind mirror law (26) $\left(\kappa_{1}=0.025\right.$, black dash-dotted line), the zero-acceleration piecewise quadratic law (25) $(\gamma=0$, black dotted line), the piecewise quadratic law (25) with the optimal static acceleration (30) (gray plain line) and the piecewise quadratic law (25) with the optimal dynamic acceleration (31) (gray dashed line). The figure represents the standard deviation of the normalized postimpact velocity $v[k] / v^{*}$ calculated over 100 impacts. Noise level is defined in (34).

output controller mirror law (26). We have tested that $\kappa_{1} \approx$ 0.025 achieves the best tradeoff between performance (rate of convergence) and robustness (noise sensitivity) in that blind mirror law.

Both the parabolic flight assumption and the Newton impact rule are perturbed by noise in an experimental setup. We simulated these perturbations by adding some noise to the reconstructed velocity $\hat{v}[k]$, whose dynamics use both the flight map and the impact rule. Equation (24) is, thus, replaced by

$$
\begin{aligned}
\hat{v}[k]=\left(e \frac{g}{2}(t[k]-t[k-1])\right. & -e \frac{s[k]-s[k-1]}{t[k]-t[k-1]} \\
& +(1+e) \dot{s}[k])\left(1+\nu[k] \epsilon_{n l}\right)
\end{aligned}
$$

where $\nu[k]$ is a random number between -1 and 1 , and $\epsilon_{n l}$ is the noise level. For the sake of illustration, we focus on the stabilization of a period-one motion, characterized by $\left(s_{\rho}^{*}, v_{\rho}^{*}\right)=$ $(0, g / 2)$, i.e., one impact per second.

Fig. 8 depicts the standard deviation of the normalized postimpact velocity $v[k] / v^{*}$ over 100 impacts, for increasing noise level. The standard deviation of the impact position does not vary significantly depending on the control law, as suggested by (28). However, the piecewise quadratic law with the acceleration tuned to cancel the dynamics in (32) [ $\gamma$ defined by (31)] achieves quasi-zero variance in postimpact velocity; see the dashed line in Fig. 8. For the tested noise levels, the standard deviation is intermediate with $\gamma=0$. In contrast, both the blind mirror law and the piecewise quadratic law with $\gamma$ defined by (30) generate twice as much variability. The excellent noise rejection obtained with $\gamma_{\text {dyn }}$ (31), even considering the nonlinear dynamics, illustrates the robustness of the piecewise quadratic control law with a suitable negative acceleration. The range of negative acceleration that produces good noise rejection is obviously limited, since the more negative value $\gamma_{\text {stat }}$ already results in poor dynamical performance. 


\section{Integral Feedback Control}

The control objective considered in the previous sections was to track the position and velocity references $\left(s_{\rho}^{*}, v_{\rho}^{*}\right)$. However, as illustrated in Section IV-B.1, it is tedious to exactly cancel the static error of the postimpact velocity in the presence of model uncertainties. Given (7), the postimpact velocity static error will result in flight time static error (i.e., pattern frequency). This static error causes a linearly growing phase shift between the reference and the actual impact times, with detrimental consequences for pattern stabilization. For example, if the frequency of impacts is not precisely assigned in the planar juggler introduced in Section II, steady-state period-two patterns will never be stabilized, since the impact frequency must be equal on each axis [see (1)].

The velocity static error can be eliminated by integral feedback: the reference trajectory is now the position $s_{\rho}[\bullet]$ and the impact time $t_{\rho}[\bullet]$ (i.e., impact phase). The corresponding velocity input $v_{\rho}[k+1]$ in $\dot{s}_{u}[k+1](25)$ is computed as the solution of (6)

$v_{\rho}[k+1]=\frac{s_{\rho}[k+2]-s_{\rho}[k+1]}{t_{\rho}[k+2]-t_{u}[k+1]}+\frac{g}{2}\left(t_{\rho}[k+2]-t_{u}[k+1]\right)$.

The difference between the reference impact time $t_{\rho}[k+2]$ and the estimated impact time $t_{u}[k+1]$ must be then constant in steady state

$$
\left(t_{\rho}[k+1]-t_{u}[k]\right)^{*}=\frac{2 v_{\rho}^{*}}{g} .
$$

Since the difference between the estimated and the actual impact times is also a constant in steady state, the delay between the reference and the actual impact times will remain constant through impacts, resulting in no static error between the desired and actual postimpact velocity and, therefore, in the pattern frequency.

Deadbeat convergence in three impacts of the integral controller is straightforwardly established by adapting the derivations of Section III-C. This controller requires reference signals two steps ahead since both $s_{\rho}[k+2]$ and $t_{\rho}[k+2]$ are used in (35).

\section{EXPERIMENTAL VALIDATION}

\section{A. Experimental Setup}

Wiper is a planar juggling robot designed from the model of the wedge billiard [see Fig. 1(a)]. Fig. 9 is a picture of

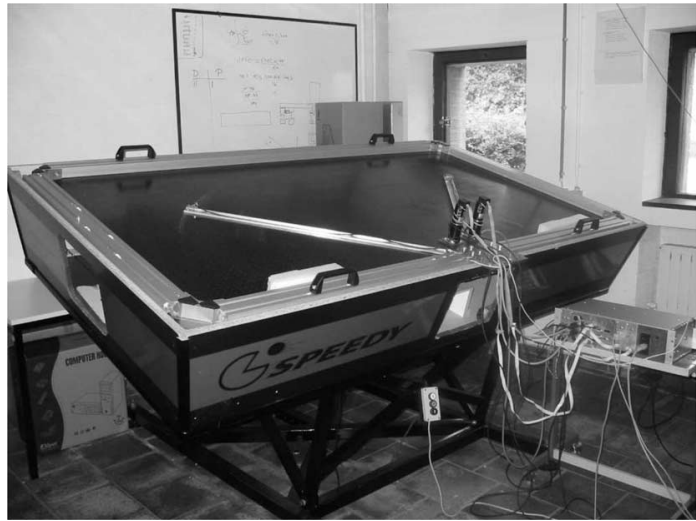

Fig. 9. Picture of Wiper.

this robot, which is called "Wiper" by analogy with the motion of windscreen wipers. The impacting edges are manufactured in aluminium, to be both light and rigid. They are actuated around their lower extremity by two independent dc motors. The motors are mounted on the rigid frame of the table, close to each other (about $10 \mathrm{~cm}$ ) since the model assumes that both edges rotate around the same point. Given the typical velocity and acceleration of the edges trajectory, we estimated the maximum rotational velocity of the motor at $4500 \mathrm{r} / \mathrm{min}$, producing a maximal torque of $55 \mathrm{mN} \cdot \mathrm{m}$. These requirements are met by the EC40-118896 motor (electronically commuted) + GP42C-203129 planetary gearhead manufactured by the Maxon Motor company (www.maxonmotor.com, Sachseln, Switzerland).

The motion plane is a tilted air-hockey table, which is pierced with a lattice of little holes, separated about $4 \mathrm{~cm}$ from each other. Constant air blowing through the holes provides the frictionless motion of the sliding puck. This puck has been made in hertalon, a nylon derivative that is both light and elastic. The gravity field $g$ can be adjusted by proper inclination of the table. The present experiments have been conducted with $12.5^{\circ}$ of table inclination. This provided comfortable cycles frequencies for experimental supervision. Moreover, the gravity constant $g$ plays no role in the stability and robustness analysis discussed previously.

The motors were actuated through two independent servoamplifiers (model DES50/5, Maxon Motor). They provided PI output control of the motors velocity, factory-designed to provide excellent static and dynamical performances. However, we superimposed a position control unit to each servoamplifier, whose

$$
\begin{gathered}
\left(\begin{array}{c}
V_{r}[k+1] \\
V_{n}[k+1]
\end{array}\right)=J(\mu[k+1])\left(\begin{array}{c}
\frac{1-\alpha^{2}}{1+\alpha^{2}} V_{r}[k]+\frac{2 \alpha^{2}}{1+\alpha^{2}}\left|V_{n}\right|[k]-g(t[k+1]-t[k]) \\
\left(\frac{-2}{1+\alpha^{2}} V_{r}[k]+\frac{1-\alpha^{2}}{1+\alpha^{2}}\left|V_{n}\right|[k]+g(t[k+1]-t[k])\right) \operatorname{sign}\left(V_{n}[k]\right)
\end{array}\right) \\
+\frac{1+e}{\alpha}\left(\begin{array}{c}
-\alpha \sin \mu[k+1] R[k+1] \\
\cos \mu[k+1] R[k+1]
\end{array}\right) \dot{\mu}[k+1] \\
R[k+1]=\frac{1+\alpha^{2}}{4 g}\left(V_{n}^{2}[k]-\left(\left|V_{n}\right|[k]-g(t[k+1]-t[k])\right)^{2}\right)
\end{gathered}
$$




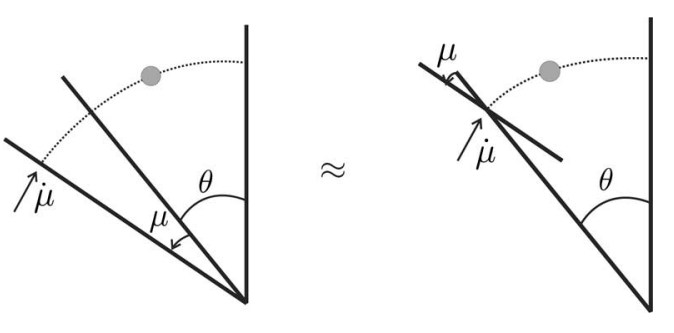

Fig. 10. Controlled rotational half-wedge (left), and the simplified model when $\mu$ is small (right).

$\mathrm{PD}^{1}$ gains were tuned to maximize the closed-loop bandwidth, which avoided inducing large vibrations in the edges during sharp transients.

The impact times are detected by two accelerometers mounted at the top of the edges to record the high-frequency small vibrations generated by the impacts. The accelerometers raw signals were band-pass filtered to remove the accelerations induced by regular edge motion (low frequencies) and noise (high frequencies). Planning of the actuation profiles, processing of the accelerometers band-pass filter, such as the motors position controller was implemented with XPCTARGET, a Simulink-oriented software (The Mathworks, Inc., Natick, MA). This software self-generated the executable code, and uploaded and executed it in a dedicated target computer, running the real-time operating system. The algorithm sampling frequency was $1 \mathrm{kHz}$.

\section{B. From the Bouncing Ball Model to the Wiper Model}

The double bouncing ball model presented in Section II is only a crude model of the Wiper robot: it neglects the coupling resulting from a rotational actuation of the edges, and a steadysate aperture possibly different from $2 \theta=90^{\circ}$. A more accurate model of the Wiper has been derived in previous work under a small angle assumption, i.e., assuming small angular deviations of the edges w.r.t. the steady-state angle $(\mu(t) \ll \theta)$, see Fig. 10 [11], [13], [24], [32], [34], [35]. Despite this simplifying assumption, the model predicted a parametric stability region of the period-one orbit, which is in excellent agreement with the experiments [24].

The Wiper model derived in [11] has three state variables$V_{r}=v_{r} / \cos \theta, V_{n}=v_{n} / \sin \theta$, and $R=r / \cos \theta$ where $v_{n}$ and $v_{r}$ are the postimpact velocities in the normal and the radial directions w.r.t. the impacted edge, and $r$ is the impact position along the edge; see Fig. 1(a), and two input variables $\mu[k]$ and $\dot{\mu}[k]$, denoting the impacted edge position w.r.t. $\theta$ (left) or $-\theta$ (right), and velocity at impact $k$. These inputs are represented in Fig. 10 for the left edge. The state-space model is given by (37), at the bottom of the previous page with the rotation matrix $J(\mu)$ :

$$
J(\mu)=\left(\begin{array}{cc}
\cos ^{2} \mu-e \sin ^{2} \mu & \frac{(1+e) \alpha}{2} \sin 2 \mu \\
\frac{1+e}{2 \alpha} \sin 2 \mu & \sin ^{2} \mu-e \cos ^{2} \mu
\end{array}\right) .
$$

\footnotetext{
${ }^{1}$ No integral gain is necessary to ensure zero static error in the output/reference transfer function, since the open-loop is an integrator: the measured output is the position while the input is a velocity signal.
}

The parameter $\alpha=\tan \theta$ depends on the wedge aperture. The flight time between two successive impacts is equal to

$$
\begin{aligned}
t[k & +1]-t[k] \\
& =\frac{1}{g}\left(\left|V_{n}^{-}\right|[k+1]+\frac{\left(\alpha^{2}-1\right)\left|V_{n}\right|[k]+2 V_{r}[k]}{1+\alpha^{2}}\right)
\end{aligned}
$$

where $\left|V_{n}^{-}\right|[k+1]$ is the preimpact normal velocity

$$
\begin{aligned}
& \left|V_{n}^{-}\right|[k+1] \\
& \quad=\sqrt{\left(\frac{2 V_{r}[k]+\left(\alpha^{2}-1\right)\left|V_{n}\right|[k]}{1+\alpha^{2}}\right)^{2}+\frac{4 g}{1+\alpha^{2}} R[k] .}
\end{aligned}
$$

The output control law (25) derived for the bouncing ball is adapted for Wiper as follows.

1) State Observer: The deadbeat velocity observer (24) is replaced by a copy of Wiper's dynamics (37), in which we substitute estimated variables $\hat{V}_{r}, \hat{V}_{n}, \hat{R}$ to the state variables $V_{r}, V_{n}, R$; and where the flight time is injected from the measurements to synchronize the observer with the actual state variables. This observer displays excellent convergence properties in simulations.

2) Tracking Controller: The tracking controller (14)-(16) is adapted to the Wiper model. The desired next impact time is estimated from (39)

$$
\begin{aligned}
& t_{u}[k+1]= \\
& t[k]+\frac{1}{g}\left(\left|\hat{V}_{n}^{-}\right|[k+1]+\frac{\left(\alpha^{2}-1\right)\left|\hat{V}_{n}\right|[k]+2 \hat{V}_{r}[k]}{1+\alpha^{2}}\right) .
\end{aligned}
$$

The position reference is simply $\mu_{\rho}^{*}=0$ since the objective is to stabilize periodic orbits of Wiper whose impacts occur at $\pm \theta$. The "local" velocity of the edge at the impact point depends obviously both on the velocity input $\dot{\mu}_{\rho}[k+1]$ and the impact radial position $R[k+1]$. Defining $\dot{S}=\dot{s} / \sin \theta$, where $\dot{s}$ is equal to this local velocity, one has $\dot{\mu}[k+1]=\alpha \dot{S}[k+1] / R[k+1]$. This "local" edge velocity derives from the impact rule

$$
\dot{S}_{u}[k+1]=\frac{\left|V_{n}\right|_{\rho}[k+1]-e\left|\hat{V}_{n}^{-}\right|[k+1]}{1+e}
$$

where the reference postimpact velocity $\left|V_{n}\right|_{\rho}[k+1]$ is equal to

$$
\begin{aligned}
\left|V_{n}\right|_{\rho}[k+1]= & \frac{g}{2 \alpha^{2}}\left(t_{\rho}[k+3]-t_{u}[k+1]\right) \\
& -\frac{g\left(1-\alpha^{2}\right)}{2 \alpha^{2}}\left(t_{\rho}[k+2]-t_{u}[k+1]\right)
\end{aligned}
$$

and is computed from the impact times reference. In the square configuration $(\alpha=1),(43)$ becomes similar to (35). In that case, the wedge-billiard dynamics decouple into two bouncing-ball dynamics, one along each edge, as mentioned in Section II.

The estimate of the radial position is obtained from the estimated state variables, according to (37) and (41)

$$
\begin{aligned}
R_{u}[k+1]= & \hat{R}[k]+\frac{1}{2 g} \hat{V}_{r}^{2}[k]+\frac{\alpha^{2}}{2 g} \hat{V}_{n}^{2}[k] \\
& -\frac{1}{2 g}\left(\left|\hat{V}_{n}\right|[k]-\hat{V}_{r}[k]-\left|\hat{V}_{n}^{-}\right|[k+1]\right)^{2} \\
& -\frac{\alpha^{2}}{2 g}\left(\hat{V}_{n}^{-}\right)^{2}[k+1]
\end{aligned}
$$


3) Continuous-Time Actuation: The piecewise quadratic control law (17) generates obviously sharp position transients at impact, even in steady state. In order to smoothen the actuation, we chose to design a closed-loop control that reaches the desired position and velocity at impact, but that is smooth in steady state. This modification helped to prevent false impact detection and motor damages in real experiments. This was realized by adapting the open-loop sinusoidal law to take feedback into account for amplitude and phase tunning

$$
\begin{aligned}
\mu(t)= & A\left(\kappa_{\mathrm{FB}} \frac{\dot{S}_{u}[k+1]}{\dot{S}^{*}} \frac{R^{*}}{R_{u}[k+1]}+\left(1-\kappa_{\mathrm{FB}}\right)\right) \\
& \times\left(\sin \left(\omega\left(t-t_{u}[k+1]\right)+\phi^{*}\right)-\sin \phi^{*}\right) \operatorname{sign}(\bullet)
\end{aligned}
$$

where $\operatorname{sign}(\bullet)=1$ for the left arm and -1 for the right one. At time $t=t_{u}[k+1]$, we obtain the desired impact position $\left(\mu\left(t_{u}[k+1]\right)=0\right)$ and the impact velocity

$$
\dot{\mu}\left(t_{u}[k+1]\right)=\kappa_{\mathrm{FB}} \dot{\mu}_{u}[k+1]+\left(1-\kappa_{\mathrm{FB}}\right) \dot{\mu}^{*}
$$

since the steady-state phase $\phi^{*}$ is given by (see [24])

$$
\phi^{*}=\arccos \left(\frac{1-e}{1+e} \frac{2 \alpha}{A} \frac{1}{\pi}\right) .
$$

The amplitude $A$ is tuned to match, in steady state, the local impact acceleration $\ddot{S}\left(t_{u}[k+1]\right)$ with the optimal value defined by (31). This acceleration is equal to $\ddot{\mu}(t)$, with $\mu$ defined in (45), and is obviously negative in steady state since $0^{\circ}<\phi^{*}<90^{\circ}$ [see (47)].

The feedback gain $0 \leq \kappa_{\mathrm{FB}} \leq 1$ is tuned to achieve the best possible tradeoff between the stabilizing performance of the observer-based output feedback controller $\left(\kappa_{\mathrm{FB}}=1\right)$ and its sensitivity to the model uncertainty.

\section{Results}

The experimental challenge was to stabilize period-one and period-two orbits of Wiper. This section describes the results that we obtained for an aperture of $\theta=40^{\circ}$. The parameters of the actuation law (45) were tuned to $A=9^{\circ}$ and $\omega=1.1 \pi \mathrm{rad} / \mathrm{s}$, while the feedback gain has been empirically tuned to $\kappa_{\mathrm{FB}}=$ 0.4 . The coefficient of restitution was estimated at about $e=$ 0.7 . The experiment initialization has been realized by proper throwing of the puck while the edges were sinusoidally actuated. Initialization then exploited the good basin of attraction of the period-one orbit in open loop. Closed-loop control was switched on after convergence of the observer. Then, the reference was switched from period-one to period-two with small aperture, and finally to period-two with large aperture. A movie is available ${ }^{2}$ to illustrate this experiment.

The flight times between two successive impacts, as detected by the accelerometers, are depicted in Fig. 11 w.r.t. the reference.

${ }^{2} \mathrm{http}: / /$ ieeexplore.ieee.org or on the first author's homepage. The material is 15.9 MB in size.

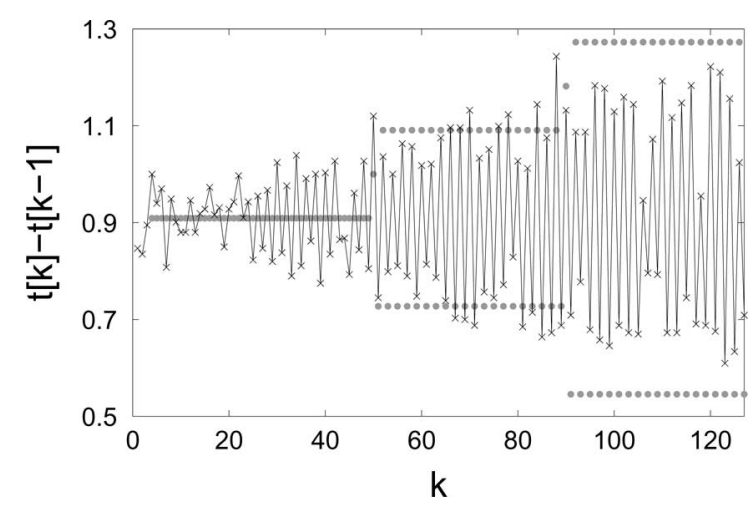

Fig. 11. Flight times between two successive impacts (detected by the accelerometers) are depicted with the black crosses. The gray circles denote the reference flight times, as defined by $t_{\rho}[k]-t_{\rho}[k-1]$. The first part of the trajectory (about $0<k<50$ ) displays the closed-loop stabilization of a period-one motion: $\Delta t^{*}=\pi / \omega \sim 0.91 \mathrm{~s}$. The second part of the trajectory (about $50<k<90$ ) displays the closed-loop stabilization of a "small" periodtwo motion, i.e., when the reference flight time alternates between $0.9 \Delta t^{*}$ and $1.1 \Delta t^{*}$. The last part of the trajectory (about $k>90$ ) displays the closed-loop stabilization of a larger period-two motion: the flight time reference alternates between $0.8 \Delta t^{*}$ and $1.2 \Delta t^{*}$.

The first part of the trajectory (till $k \simeq 50$ ) displays the closedloop stabilization of the period-one motion, i.e., when the target flight time is always equal to $\Delta t^{*}=\pi / \omega \simeq 0.91 \mathrm{~s}$. The second part of the trajectory (about $50<k<90$ ) displays the closedloop stabilization of a "small" period-two motion, i.e., when the flight time alternates between 0.9 and $1.1 \Delta t^{*}$. The last part of the trajectory (about $k>90$ ) displays the closed-loop stabilization of a larger period-two motion: the flight time reference alternates between 0.8 and $1.2 \Delta t^{*}$. The mismatch between the reference and the actual trajectory increases for the "large" period-two orbit. This can be explained as follows: the steady-state velocity $\dot{S}^{*}$ and impact position $R^{*}$ depend on the reference pattern [24]. However, for the sake of simplicity, we let them equal to the steady-state values of the period-one motion in the control law (45), regardless of the reference. This introduces a steady-state error w.r.t. the two reference flight times when they significantly differ from each other, since $\alpha \neq 1$.

This successful experimental validation of period-two orbits in Wiper, with robust control of the trajectory, contrasts with previous results obtained with a sensorless control [24]. In that case, indeed, the period-two orbits collapsed after a few seconds, due to their small basins of attraction. This illustrates that feedback is required to stabilize the shower pattern with Wiper, but with limited sensing demand since a robust control law can be designed from the impact times information only. In agreement with the robustness analysis of Section V, we mention that sustained stabilization in the laboratory required a proper (negative) tuning of the impact acceleration and could never be achieved with positive acceleration.

\section{CONCLUSION}

This paper presents the design, analysis, and experimental validation of robust closed-loop control of two periodic patterns in a planar juggler. The control law only uses the impact times as feedback information, thereby, relaxing the need for complex 
sensor design. The parameter $\gamma$, i.e., the acceleration at impact, plays no role in the stability analysis. In contrast, proper tuning of this parameter was shown to have a dramatic effect on robustness and to be a key to success for the experimental validation. Robustness requires negative acceleration, in accordance with sensorless control strategies [5], [24] and with observed human strategies [16]-[18]. This is in contrast with the mirror law algorithm proposed earlier in the literature [25]-[27] and possibly explains why such control schemes - that have been shown to perform robustly in 1-D, 2-D, and even 3-D environments with a continuous-time sensing of the juggled object-may perform poorly in implementations with limited sensing capabilities. The paper illustrated that the measurement of impact times is both a cheap and relevant feedback source in juggling experiments. It would be interesting to investigate whether this may supplement the continuous-time sensing required in more complicated juggling implementations, e.g., in 3-D environments.

\section{APPENDIX I}

\section{Stability of the Period-One Pattern with Sinusoidal Actuation}

Stability of the period-one pattern with sinusoidal actuation is studied via the linearization of (4) and (5), considering $s(t)=A \sin (\omega t)$. It has been first derived in [5] and [6], then in [31], relaxing the so-called small amplitude assumption. This appendix reviews the derivations of [31].
Steady-state ball postimpact velocity $v^{*}$, impact phase $\phi^{*}$, and position $s^{*}$ are given by

$$
\begin{aligned}
& v^{*}=\frac{\pi n g}{\omega} \\
& \dot{s}^{*}=A \omega \cos \phi^{*}=\frac{(1-e) \pi n g}{(1+e) \omega} \\
& s^{*}=A \sin \phi^{*}=A \sqrt{1-\left(\frac{(1-e) \pi n g}{(1+e) A \omega^{2}}\right)^{2}} .
\end{aligned}
$$

Linearizing (4) and (5) around the steady state (51) gives

$$
\delta t[k+1]=\delta t[k]+\frac{1+e}{g} \delta v[k]
$$

$$
\begin{aligned}
\delta v[k+1]= & e^{2} \delta v[k]-(1+e) A \omega^{2} \\
& \sqrt{1-\left(\frac{(1-e) \pi n g}{(1+e) A \omega^{2}}\right)^{2}} \delta t[k+1]
\end{aligned}
$$

where $\delta t$ and $\delta v$ denote the first-order small perturbations on the impact time and postimpact velocity, respectively. These equations can be written with the nondimensional matrix form (48), given at the bottom of this page.

$$
\begin{aligned}
& \left(\begin{array}{c}
\frac{\omega}{g} \delta v[k+1] \\
\omega \delta t[k+1]
\end{array}\right)=\left(\begin{array}{cc}
e^{2} & 0 \\
1 & 1+e
\end{array}\right)\left(\begin{array}{c}
\frac{\omega}{g} \delta v[k] \\
\omega \delta t[k]
\end{array}\right)+\left(\begin{array}{c}
-(1+e) \frac{A \omega^{2}}{g} \sqrt{1-\left(\frac{(1-e) \pi n g}{(1+e) A \omega^{2}}\right)^{2}} \\
0
\end{array}\right) \omega \delta t[k+1] \\
& =(\underbrace{\left(\begin{array}{cc}
e^{2} & 0 \\
1 & 1+e
\end{array}\right)}_{\mathbf{A}}+\underbrace{\left(\begin{array}{c}
-(1+e) \frac{A \omega^{2}}{g} \sqrt{1-\left(\frac{(1-e) \pi n g}{(1+e) A \omega^{2}}\right)^{2}} \\
0
\end{array}\right)}_{\mathbf{B}} \underbrace{\left(\begin{array}{ll}
1 & 1+e)
\end{array}\right)}_{\mathbf{C}}\left(\begin{array}{c}
\frac{\omega}{g} \delta v[k] \\
\omega \delta t[k]
\end{array}\right) \\
& \delta s[k+1]=\delta s[k]+\frac{2 \pi n}{\omega} \delta v[k]-\frac{\pi n g}{\omega}(\delta t[k+1]-\delta t[k]) \\
& \delta v[k+1]=-e \delta v[k]+e g(\delta t[k+1]-\delta t[k])+(1+e) \delta \dot{s}[k+1]+\frac{2}{1+e} \frac{\pi n g}{\omega} \delta e[k+1] \\
& \delta s[k+1]=\frac{2 e}{1+e} \delta s_{\rho}[k+1]+\frac{1-e}{1+e} \delta s[k]+\frac{1-e}{1+e} \frac{\pi n g}{\omega}(\delta t[k+1]-\delta t[k])-\frac{1-e}{1+e} \frac{2 \pi n}{\omega} \delta \hat{v}[k] \\
& \delta \dot{s}[k+1]=\frac{1}{1+e} \delta v_{\rho}[k+1]-\left(\frac{e}{1+e}+\frac{2 \gamma}{g}\right) \delta \hat{v}[k]+\left(\frac{e}{1+e}+\frac{\gamma}{g}\right) \frac{\omega}{\pi n}\left(\delta s_{\rho}[k+1]-\delta s[k]\right)+\gamma(\delta t[k+1]-\delta t[k]) \\
& \delta \hat{v}[k+1]=e \frac{g}{2}(\delta t[k+1]-\delta t[k])-e \frac{\omega}{2 \pi n}(\delta s[k+1]-\delta s[k])+(1+e) \delta \dot{s}[k+1] \\
& \left(\begin{array}{c}
\frac{g \delta s[k+1]}{\left(v_{\rho}^{*}\right)^{2}} \\
\frac{\delta v[k+1]}{v_{\rho}^{*}} \\
\frac{g \delta t[k+1]}{v_{\rho}^{*}} \\
\frac{\delta \hat{v}[k+1]}{v_{\rho}^{*}}
\end{array}\right)=\underbrace{\left(\begin{array}{cccc}
0 & 1-e & 0 & -(1-e) \\
0 & e^{2}+\frac{\gamma}{g}(1+e)^{2} & 0 & -\left(e^{2}+\frac{\gamma}{g}(1+e)^{2}\right) \\
1 & 1+e & 1 & 1-e \\
0 & e^{2}+\frac{\gamma}{g}(1+e)^{2} & 0 & -\left(e^{2}+\frac{\gamma}{g}(1+e)^{2}\right)
\end{array}\right)}_{\mathbf{A}^{\prime}}\left(\begin{array}{c}
\frac{g \delta s[k]}{\left(v_{\rho}^{*}\right)^{2}} \\
\frac{\delta v[k]}{v_{\rho}^{*}} \\
\frac{g \delta t[k]}{v_{\rho}^{*}} \\
\frac{\delta \hat{v}[k]}{v_{\rho}^{*}}
\end{array}\right)+\underbrace{\left(\begin{array}{ccc}
1 & 0 & 0 \\
0 & 1 & \frac{2}{1+e} \\
-1 & 0 & 0 \\
0 & 0
\end{array}\right)}_{\mathbf{B}^{\prime}}\left(\begin{array}{c}
\frac{g \delta s_{\rho}[k+1]}{\left(v_{\rho}^{*}\right)^{2}} \\
\frac{\delta v_{\rho}[k+1]}{v_{\rho}^{*}} \\
\delta e[k+1]
\end{array}\right)
\end{aligned}
$$


It can be shown that the eigenvalues of $(\mathbf{A}+\mathbf{B C})$ lie into the unitary circle if and only if the following condition holds [31]:

$$
\pi n \frac{1-e}{1+e}<\frac{A \omega^{2}}{g}<\sqrt{\pi^{2} n^{2}\left(\frac{1-e}{1+e}\right)^{2}+\frac{4\left(1+e^{2}\right)^{2}}{(1+e)^{4}}} .
$$

This corresponds to the amplitude and frequency range of stability for the period-one motion.

\section{APPENDIX II}

\section{Linearized Equations of the 1-D Noisy Bouncing Ball with Piecewise Quadratic Control}

The linearized dynamics of the perturbed 1-D bouncingball dynamics (4) and (27), and the piecewise quadratic law (25) are given in (49), at the bottom of the previous page, where $\delta e[k+1]$ is the small perturbation on the coefficient of restitution and is considered as an additional input. Using nondimensional state variables, one obtains the state-space model (50), given at the bottom of the previous page. The state variables are small perturbations of the ball impact position $g \delta s[k+1] /\left(v_{\rho}^{*}\right)^{2}$ and velocity $\delta v[k+1] / v_{\rho}^{*}$; the impact time $g \delta t[k+1] / v_{\rho}^{*}$ and the observed velocity $\delta \hat{v}[k+1] / v_{\rho}^{*}$.

The matrix $\mathbf{A}^{\prime}$ is singular. This is a consequence of deadbeat convergence of the 1-D bouncing ball, controlled with the piecewise quadratic law (25).

\section{ACKNOWLEDGMENT}

The authors would like to thank Manuel Gérard for his contributions to the development of Wiper and Michel De Wan for his precious technical assistance during the design of this robot. They would also like to thank the reviewers for their comments that helped to improve the paper.

\section{REFERENCES}

[1] A. Cohen, S. Rossignol, and S. Grillner, Neural Control of Rhythmic Movements in Vertebrates. New York: Wiley, 1988.

[2] K. Pearson, "Motor systems," Curr. Opin. Neurobiol., vol. 10, no. 5, pp. 649-654, 2000.

[3] S. Schaal, D. Sternad, R. Osu, and M. Kawato, "Rhythmic arm movement is not discrete," Nat. Neurosci., vol. 7, no. 10, pp. 1136-1143, 2004.

[4] S. Schaal and N. Schweighofer, "Computational motor control in humans and robots," Curr. Opin. Neurobiol., vol. 15, no. 6, pp. 675-682, 2005.

[5] P. J. Holmes, "The dynamics of repeated impacts with a sinusoidally vibrating table," J. Sound Vibr., vol. 84, no. 2, pp. 173-189, 1982.

[6] J. Guckenheimer and P. J. Holmes, Nonlinear Oscillations, Dynamical Systems and Bifurcations of Vector Fields. New York: Springer-Verlag, 1986.

[7] A. Tornambe, "Modeling and control of impact in mechanical systems: Theory and experimental results," IEEE Trans. Autom. Control, vol. 44, no. 2, pp. 294-309, Feb. 1999.

[8] K. M. Lynch and C. K. Black, "Recurrence, controllability, and stabilization of juggling," IEEE Trans. Robot. Autom., vol. 17, no. 2, pp. 113-124, Apr. 2001.

[9] M. W. Spong, "Impact controllability of an air hockey puck," Syst. Control Lett., vol. 42, no. 5, pp. 333-345, 2001.

[10] A. Zavala-Rio and B. Brogliato, "Direct adaptive control design for one-degree-of-freedom complementary-slackness jugglers," Automatica, vol. 37, no. 7, pp. 1117-1123, 2001.
[11] R. Sepulchre and M. Gerard, "Stabilization of periodic orbits in a wedge billiard," in Proc. 42nd IEEE Conf. Decision Control, 2003, pp. 15681573.

[12] L. Menini and A. Tornambe, "Control of (otherwise) uncontrollable linear mechanical systems through non-smooth impacts," Syst. Control Lett., vol. 49, no. 4, pp. 311-322, 2003.

[13] M. Gerard and R. Sepulchre, "Stabilization through weak and occasional interactions: A billiard benchmark," in Proc. 6th IFAC Symp. Nonlinear Control Syst., Stuttgart, Germany, 2004, pp. 75-80.

[14] B. Brogliato, M. Mabrouk, and A. Zavala-Rio, "On the controllability of linear juggling mechanical systems," Syst. Control Lett., vol. 55, pp. 350367, 2006.

[15] H. Hirai and F. Miyazaki, "Dynamic coordination between robots: Selforganized timing selection in a juggling-like ball-passing task," IEEE Trans. Syst. Man Cybern. B, Cybern., vol. 36, no. 4, pp. 738-754, Aug. 2006.

[16] S. Schaal, C. G. Atkeson, and D. Sternad, "One-handed juggling: A dynamical approach to a rhythmic movement task," J. Mot. Behav., vol. 28, no. 2, pp. 165-183, 1996.

[17] D. Sternad, M. Duarte, H. Katsumata, and S. Schaal, "Dynamics of a bouncing ball in human performance," Phys. Rev. E, vol. 6301, no. 1 , pp. 011902-1-011902-1-8, 2001.

[18] D. Sternad, M. Duarte, H. Katsumata, and S. Schaal, "Bouncing a ball: Tuning into dynamic stability," J. Exp. Psychol. Hum. Percept. Perform. vol. 27, no. 5, pp. 1163-1184, 2001.

[19] C. E. Shannon, "Scientific aspects of juggling," in Claude Elwood Shannon: Collected Papers, N. J. A. Sloane and A. D. Wyner, Eds. New York: IEEE, 1993, p. 924.

[20] U. Saranli, M. Buehler, and D. Koditschek, "Rhex-A simple and highly mobile hexapod robot," Int. J. Robot. Res., vol. 20, no. 7, pp. 616-631, 2001.

[21] P. Holmes, R. J. Full, D. Koditschek, and J. Guckenheimer, "The dynamics of legged locomotion: Models, analyses, and challenges,"Siam Rev. vol. 48, no. 2, pp. 207-304, 2006

[22] E. R. Westervelt, G. Buche, and J. W. Grizzle, "Experimental validation of a framework for the design of controllers that induce stable walking in planar bipeds," Int. J. Robot. Res., vol. 23, no. 6, pp. 559-582, 2004.

[23] E. L. Amazeen, P. G. Amazeen, A. A. Post, and P. J. Beek, "Timing the selection of information during rhythmic catching," J. Mot. Behav., vol. 31, no. 3, pp. 279-289, 1999.

[24] R. Ronsse, P. Lefevre, and R. Sepulchre, "Sensorless stabilization of bounce juggling," IEEE Trans. Robot., vol. 22, no. 1, pp. 147-159, Feb. 2006.

[25] M. Buehler, D. Koditschek, and P. Kindlmann, "A one degree of freedom juggler in a two degree of freedom environment," in IEEE/RSJ Conf. Intell. Syst. Robots, Tokyo, Japan, 1988, pp. 91-97.

[26] M. Buehler, D. Koditschek, and P. Kindlmann, "A family of robot control strategies for intermittent dynamical environments,"IEEE Control Syst. Mag., vol. 10, no. 2, pp. 16-22, 1990.

[27] M. Buehler, D. Koditschek, and P. Kindlmann, "Planning and control of robotic juggling and catching tasks," Int. J. Robot. Res., vol. 13, no. 2, pp. 101-118, 1994.

[28] B. Brogliato and A. Zavala-Rio, "On the control of complementaryslackness juggling mechanical systems," IEEE Trans. Autom. Control, vol. 45, no. 2, pp. 235-246, Feb. 2000.

[29] T. L. Vincent and A. I. Mees, "Controlling a bouncing ball," Int.J. Bifurcat Chaos, vol. 10, no. 3, pp. 579-592, 2000.

[30] R. Huys and P. J. Beek, "The coupling between point-of-gaze and ball movements in three-ball cascade juggling: The effects of expertise, pattern and tempo," J. Sports Sci., vol. 20, no. 3, pp. 171-186, 2002.

[31] C. Bapat, S. Sankar, and N. Popplewell, "Repeated impacts on a sinusoidally vibrating table," J. Sound Vibr., vol. 108, no. 1, pp. 99-115, 1986.

[32] R. Ronsse, P. Lefevre, and R. Sepulchre, "Timing feedback control of a rhythmic system," in Proc. 44th IEEE CDC/ECC 2005, Seville, Spain, 2005, pp. 6146-6151.

[33] R. Ronsse and R. Sepulchre, "Feedback control of impact dynamics: The bouncing ball revisited," in Proc. 45th IEEE Conf. Control, San Diego, CA, 2006, pp. 4807-4812.

[34] H. E. Lehtihet and B. N. Miller, "Numerical study of a billiard in a gravitational field," Phys. D, vol. 21, no. 1, pp. 93-104, 1986.

[35] M. Gerard and R. Sepulchre, "Rhythmic stabilization of periodic orbits in a wedge," in Proc. IFAC World Congr., Prague, Czech Republic, 2005. 


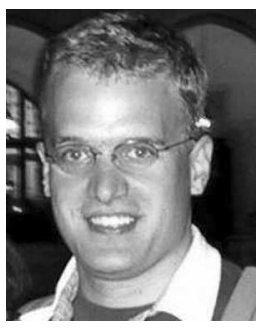

Renaud Ronsse was born in Liège, Belgium, in 1980. He received the University degree in electrical engineering from the Université de Liège, Liège, in 2002, where he is currently working toward the Ph.D. degree at the Department of Electrical Engineering and Computer Science.

His current research interests include the control of rhythmic tasks, emphasizing the correspondences between efficient feedback algorithms for robotics and the human behavior.

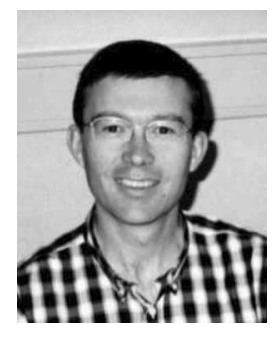

Philippe Lefèvre was born in Ottignies, Belgium, in 1965. He received the University degree in electrical engineering and the Ph.D. degree in biomedical engineering from the Université Catholique de Louvain (UCL), Louvain-la-Neuve, Belgium, in 1988 and 1992, respectively.

From 1995 to 1997, he was a Postdoctoral Researcher with the Laboratory of Sensorimotor Research, National Institutes of Health (NIH), Bethesda, MD. In 1997, he obtained a permanent position at the Fonds de la Recherche Scientifique (FNRS), UCL, where he was engaged in investigating the neural control of movement by combining both experimental and modeling approaches. From 2003 to 2004, he was a Visiting Scientist with the National Eye Institute, NIH. He is currently with the Center for Systems Engineering and Applied Mechanics (CESAME), UCL.

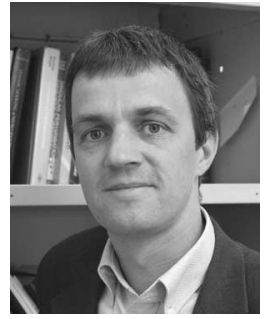

Rodolphe Sepulchre was born in Brussels, Belgium, in 1967. He received the University and Ph.D. degrees in electrical engineering science (applied mathematics) from the Université Catholique de Louvain, Louvain-la-Neuve, Belgium, in 1990 and 1994, respectively.

From 1994 to 1996, he was a visiting Researcher with the University of California, Santa Barbara, and with Princeton University, Princeton, NJ, from 2002 to 2003. He is currently a Professor with the Université de Liège, Liège, Belgium. His current research interests include nonlinear dynamics and control, and their applications to problems in mechanical, electrical, and biomedical engineering. He is the coauthor of the book Constructive Nonlinear Control (Springer, 1997). 